\title{
Síntese e caracterização de novos copolímeros fosforilados
}

\section{Synthesis and characterization of new phosphorylated copolymers}

\author{
Viviane Martins Rebello dos Santos ${ }^{1}$, Katia Novack Monteiro', Denise Versiane Monteiro de Sousa ${ }^{1}$, \\ Sheila Rodrigues Oliveira² e Claudio Luis Donnici²
}

${ }^{1}$ Departamento de Química, Instituto de Ciências Exatas e Biológicas, Universidade Federal de Ouro Preto - UFOP, Ouro Preto, MG, Brasil

${ }^{2}$ Departamento de Química, Universidade Federal de Minas Gerais - UFMG, Belo Horizonte, MG, Brasil

*vivianesantos@iceb.ufop.br

\begin{abstract}
Resumo
O rápido desenvolvimento da química de polímeros nos últimos anos tem acompanhado a introdução dos materiais poliméricos no campo da medicina. Polímeros com aplicações médicas podem ser subdivididos em dois grandes grupos: materiais usados em próteses e a produção de polímeros biologicamente ativos. Neste trabalho foram sintetizados copolímeros possuindo cadeia principal de poli (metacrilato de metila) e cadeias enxertadas de polietilenoglicol (COP GRAFT). A partir da síntese do copolímero enxertado inicial foram realizadas modificações em suas cadeias de modo a aumentar a possibilidade de incorporação de um fármaco que será utilizado em sistema de liberação controlada de medicamentos. Os novos compostos foram obtidos através de duas rotas sintéticas e os produtos formados ao final de cada etapa foram purificados e caracterizados. Através da técnica de infravermelho (FITIR) foi possível observar as bandas de absorções características das ligações $\mathrm{P}-\mathrm{H}, \mathrm{P}=\mathrm{O}$ e P-O, que foram inseridas na cadeia do COP GRAFT durante a fosforilação, além das bandas de absorções características do copolímero, antes da fosforilação, como C=O e C-O. A caracterização térmica das amostras utilizando termogravimetria (TGA) e calorimetria diferencial de varredura (DSC) mostrou que apenas o copolímero de diisobutila apresentou temperatura de transição endotérmica e comportamentos de degradação compatíveis a de um polímero. Através da técnica de difração de raios X (XRD) foi possível observar uma modificação da cristalinidade das amostras, provavelmente devido a uma variação da conformação das cadeias após a inserção de novos grupos químicos. Nos cromatogramas obtidos pela técnica de cromatografia de permeação em gel (GPC) percebeu-se aumento de massa após a fosforilação com o grupo diisobutila no COPGRAFT. A reação de fosforilação foi eficiente na obtenção do copolímero de diisobutila e as técnicas aplicadas demonstraram que houve a modificação no copolímero enxertado.
\end{abstract}

Palavras-chave: sintese e modificação de copolímero, fosforilação, caracterização por FTIR, DSC, TGA, XRD e GPC.

\begin{abstract}
The rapid development in polymer chemistry has, in recent years, led to the introduction of polymeric materials in the field of medicine. Two major groups of polymers are used for medical applications: prosthetic and biologically active materials. We synthesized copolymers with the main chain consisting of poly (methyl methacrylate) and graft chains of poly(ethylene glycol) (COP GRAFT). Changes were made in the graft copolymer chains for a possible incorporation of a drug, such that it may be used for the controlled release of drugs. The synthesis of these new compounds is based on two synthetic routes and FTIR, differential scanning calorimetry (DSC), thermogravimetry (TGA) and X-ray diffraction (XRD) characterized the products formed at the end of each stage. Through FTIR we observed absorption bands characteristic of $\mathrm{P}-\mathrm{H}, \mathrm{P}=\mathrm{O}$ and $\mathrm{P}-\mathrm{O}$ bonds that were inserted into the polymer chain during phosphorylation of the COP GRAFT, beyond the absorption bands characteristic of the copolymer before phosphorylation, such as $\mathrm{C}=\mathrm{O}$ and C-O. By TGA and DSC we observed that only the diisobutyl copolymer has endothermic transition temperature and degradation behavior consistent with a polymer. XRD results showed a structural and conformational change in crystallinity of modified chains. Molecular weight and polydispersity of COPGRAFT obtained by GPC increased after phosphorylation with diisobutyl group. The phosphorylation reaction was efficient in obtaining diisobutyl copolymer and the applied techniques confirmed the modification of the COPGRAFT chains.
\end{abstract}

Keywords: synthesis and modification of copolymer, phosphorylation, characterization by FTIR, DSC, TGA, XRD and GPC. 


\section{Introdução}

Os polímeros, tanto os naturais como os sintéticos, são uma das classes de materiais mais versáteis e têm mudado nosso cotidiano por várias décadas com importantes aplicações principalmente nas áreas médica e farmacêutica ${ }^{[1,2]}$.

A atividade biológica de um polímero é determinada pela capacidade intrínseca do mesmo em interagir direta ou indiretamente com outras moléculas ou sítios enzimáticos por meio de interações físicas e/ou químicas e produzindo por meio delas uma bio-resposta. $\mathrm{O}$ fármaco pode fazer parte do esqueleto da cadeia polimérica ou ser ligado a uma cadeia lateral. Uma vez que estes fármacos macromoleculares têm sua concentração aumentada em solução, acabam por atingir o efeito desejado com uma menor dosagem. Polímeros com fármacos ativos como parte da cadeia principal são usualmente preparados pela associação de fármacos comuns com polímeros comerciais ou levemente modificados. Os polímeros empregados nesse estudo foram selecionados devido às suas propriedades físicas: transparência e resistência do metacrilato de metila (MMA), estrutura apropriada e fácil processamento do polietilenoglicol (PEG). O polímero a ser empregado nas formulações de liberação controlada de fármacos deve ser quimicamente inerte, ou seja, não apresentar antigenicidade intrínseca; ser preferencialmente biodegradável, livre de impurezas, não acumular-se no organismo, apresentar grupos funcionais para ligação química e, principalmente, manter a atividade original do fármaco liberado até que atinja o local de ação ${ }^{[3,4]}$. A combinação fármaco-polímero pode ocorrer através de ligações covalentes, ligações iônicas ou ainda por forças de dipolos. A natureza da ligação fármaco-suporte está relacionada com o local de administração. Deste modo, ligações covalentes são recomendáveis na administração de fármacos por via oral. Em casos onde existem outros tipos de ligação, podem ocorrer alterações na cadeia polimérica a medida que a mesma circula por diferentes locais do corpo: isso acarretaria dramáticas mudanças na atividade biológica $^{[3,5,6]}$.

É grande o número de materiais de origem polimérica utilizados na liberação controlada de fármacos e outros agentes ativos. Os primeiros polímeros empregados foram selecionados, apesar de seu uso não biológico, mas sim devido às suas propriedades físicas, como a elasticidade dos poliuretanos e a resistência física e transparência do poli(metacrilato de metila $)^{[7]}$. O PEG, por sua vez, vem sendo muito empregado como biopolímero no tratamento anticâncer. O interesse crescente na modificação de polímeros através de ligações químicas com outra espécie polimérica se baseia no fato de que os copolímeros graftizados resultantes apresentam propriedades únicas, não encontradas nos homopolímeros correspondentes ${ }^{[6,8,9]}$. Devido a essas características o copolímero sintetizado faz parte da classe dos polímeros farmacologicamente ativos que são macromoléculas que podem agir como medicamentos ou podem ser usados como veículos para outros fármacos ${ }^{[9]}$.

Devido à grande reatividade do átomo de fósforo e da grande aplicação de compostos fosforados principalmente na medicina como antitumorais ou antineoplásicos ${ }^{[10-13]}$ este trabalho objetiva primeiramente sintetizar e caracterizar um copolímero graftizado a partir do metacrilato de metila e polietileno glicol (COP GRFT), de modo a obter um material que reúna as propriedades físicas e químicas dos dois polímeros e, posteriormente, alterar as propriedades e comportamento desse copolímero através de reações de fosforilação, aumentando assim sua combinação com possíveis fármacos, favorecendo uma eficaz liberação desses fármacos para aplicação tanto em animais e em seres humanos quanto na agricultura. Posteriormente será verificada a possibilidade desse copolímero ser utilizado como matriz para inserção de agente quimioterápico ${ }^{[14,10-13]}$.

\section{Experimental}

\subsection{Reagentes químicos}

O polietilenoglicol (PEG) comercial foi utilizado como recebido; o metacrilato de metila (MMA) com grau de pureza 99\%, o peróxido de benzoíla com grau de pureza $98 \%$ e o fosfito de diisopropila com grau de pureza $97,0 \%$ foram fornecidos pela Sigma-Aldrich; o diclorometano com grau de pureza 99,5\%, o álcool etílico com grau de pureza $95 \%$ e o tetracloreto de carbono com grau de pureza 99,9\% foram fornecidos pela VETEC; o hidróxido de sódio P.A. foi fornecido pela Reagen. Os reagentes fosfito de dipropila, fosfito de dibutila, fosfito de diisobutila, fosfito de diisopropila e fosfito de diisopentila foram sintetizados pelo nosso grupo de pesquisa ${ }^{[10-12]}$.

\subsection{Síntese do copolímero graftizado ${ }^{[15,16]}$}

A obtenção do copolímero graftizado foi realizada a partir da adaptação da metodologia descrita segundo Novack e Oliveira $^{[17]}$. O copolímero foi preparado em béquer a partir da dissolução de 40,00g de polietilenoglicol em 100,00 mL de diclorometano. O conteúdo do béquer foi transferido para um balão de fundo redondo juntamente com $2,80 \mathrm{~mL}$ de MMA e 0,05 g do iniciador peróxido de benzoíla. Em seguida, a mistura reacional foi deixada sob agitação magnética à temperatura de $80^{\circ} \mathrm{C}$ por 6 horas. Ao término da reação foram adicionados $50,00 \mathrm{~mL}$ de diclorometano e $50,00 \mathrm{~mL}$ de água destilada, gerando uma solução homogênea incolor. O conteúdo do balão foi transferido para um funil de separação e a fase orgânica separada da fase aquosa com diclorometano. O copolímero (COP GRAFT) obtido na reação foi transferido para um béquer e deixado à temperatura ambiente para evaporar o solvente e solidificar-se, conforme a $1^{\mathrm{a}}$ etapa da Figura 1.

\subsection{Síntese e purificação do copolímero fosforilado ${ }^{[11-13]}$}

A fosforilação do copolímero foi realizada com cinco fosfitos de diferentes grupos alquilas (fosfito de dipropila, diisopropila, dibutila, diisobutila e diisopentila $)^{[11]}$, conforme a $2^{\mathrm{a}}$ etapa da Figura 1. Em um béquer foi dissolvido 1,00 g do COP GRAFT em diclorometano sendo em seguida adicionado a esse sistema uma mistura de 10,00 $\mathrm{mL}$ de água destilada e $0,15 \mathrm{~g}$ de $\mathrm{NaOH}$. A mistura foi deixada sob agitação por $20 \mathrm{~min}$. Em outro béquer foi preparada uma mistura de $1,00 \mathrm{~g}$ do fosfito de dialquila e $0,59 \mathrm{~mL}$ de $\mathrm{CCl}_{4}$, que foi deixada sob agitação por $20 \mathrm{~min}$. Em um balão de fundo redondo equipado com um condensador de refluxo, foram adicionadas as misturas anteriormente preparadas $\mathrm{e}$ o sistema foi mantido sob banho-maria à $40^{\circ} \mathrm{C}$ durante $4 \mathrm{~h}$. 
Ao término da reação foi feita a purificação do produto pela técnica de extração líquido-líquido utilizando diclorometano. O copolímero fosforilado obtido na reação foi transferido para um béquer e deixado à temperatura ambiente para evaporação do solvente. A purificação do copolímero foi realizada utilizando a técnica de cromatografia em coluna utilizando como fase estacionária sílica gel e fase móvel uma mistura eluente de $70 \%$ diclorometano e $30 \%$ hexano, a fim de eliminar impurezas, principalmente reagentes de partida e subprodutos provenientes de oxidações ${ }^{[18]}$.

\subsection{Caracterização}

\subsubsection{Análise por espectrometria na região do Infravermelho com} Transformada de Fourier (FTIR)

A técnica de FTIR foi utilizada para caracterizar o COP GRAFT e os copolímeros fosforilados sintetizados. Os espectros foram obtidos utilizando o equipamento IV Nicolet 380 FT-IR, modelo ThermoScientific, na faixa de número de onda compreendida entre $500-4000 \mathrm{~cm}^{-1} \mathrm{e}$ resolução de $4 \mathrm{~cm}^{-1}$. Os espectros foram normalizados e as principais bandas de vibração foram associadas com grupos químicos. Estes espectros foram reproduzidos através do programa Ezomnic.

\subsubsection{Análise Termogravimétrica (TGA)}

O copolímero graftizado e os copolímeros fosforilados foram avaliados por análises termogravimétricas em um equipamento da TA Instruments, modelo SDT 2960 Simultaneous DTA-TGA, empregando uma taxa de aquecimento de $20^{\circ} \mathrm{C} / \mathrm{min}$ de 20 a $700^{\circ} \mathrm{C}$ e sob atmosfera de nitrogênio e fluxo $100 \mathrm{~mL} / \mathrm{min}$. Foram utilizados cadinhos de alumina.

\subsubsection{Calorimetria Diferencial de Varredura (DSC)}

As análises de DSC dos copolímeros graftizado e fosforilados foram conduzidas em um equipamento da TA Instruments, modelo DSC 2010, usando cadinhos de

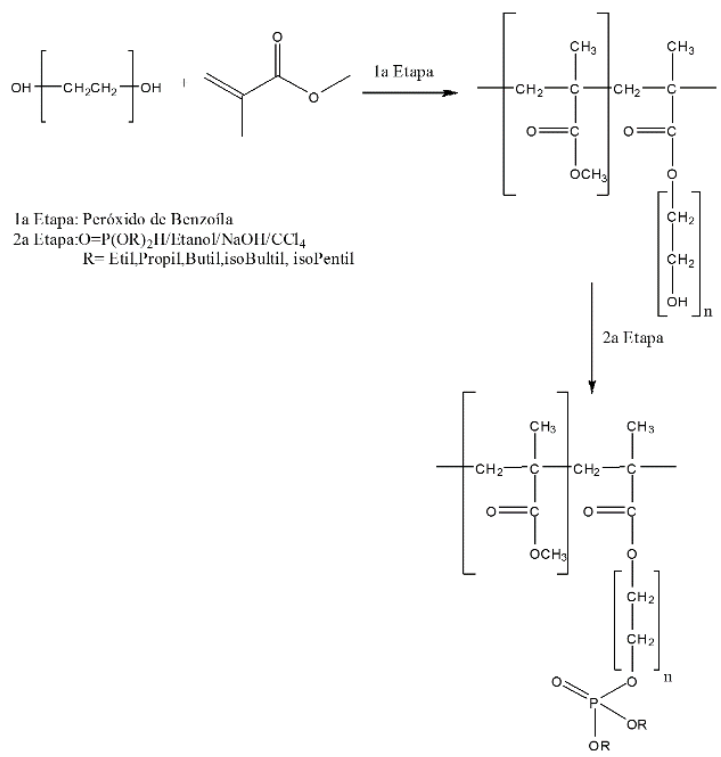

Figura 1. Síntese do copolímero graftizado PMMA-g-PEG e derivados fosforilados. alumínio e operando a uma taxa de $20^{\circ} \mathrm{C} / \mathrm{min}$, no intervalo de 20 a $350^{\circ} \mathrm{C}$ sob atmosfera de nitrogênio, com fluxo de $70 \mathrm{~mL} / \mathrm{min}$.

\subsubsection{Análise por difração de raios X (XRD)}

Os difratogramas de raios $\mathrm{X}$ foram obtidos à temperatura ambiente e foram utilizados filmes ou pós-prensados em porta amostras de alumínio. As amostras foram analisadas no intervalo de ângulos de difração $2 \Theta$ variando de 5 a $70^{\circ} \mathrm{e}$ velocidade $2^{\circ} \mathrm{C} / \mathrm{mim}^{-1}$. As análises foram realizadas em um difratômetro Shimadzu, modelo XRD-6000, equipado com tubo de ferro e monocromador de grafite.

\subsubsection{Cromatografia de permeação em gel (GPC)}

As massas molares numérica média $(\mathrm{Mn})$, ponderal média em massa (Mw) e o índice de polidispersão (IP) dos polímeros em análise foram determinados por cromatografia de permeação em gel (GPC) usando um cromatógrafo provido de três colunas de ultrastyragel linear e $500 \mathrm{~A}^{\circ}$, operando como fase móvel o solvente THF (tetraidrofurano) e um volume de injeção de $10,00 \mathrm{~mL} / \mathrm{min}$ num tempo total de $35 \mathrm{~min}$.

\section{Resultados e Discussões}

A síntese do copolímero graftizado apresentou um produto incolor, amorfo e rígido, com rendimento de $93,4 \%$, conforme Figura 1.

Nas reações de fosforilação do COP GRAFT foi necessária a seleção da condição de reação mais eficiente para evitar perda de reagentes e com isso à fosforilação foi realizada em tempos de reações variados. Num tempo de reação de $6 \mathrm{~h}$ o produto fosforilado apresentou no espectro de FTIR bandas de absorções referentes ao estiramento de $\mathrm{O}-\mathrm{H}$ em torno de $3400 \mathrm{~cm}^{-1}$, que expressa à presença do COP GRAFT ou presença de $\mathrm{H}_{2} \mathrm{O}$. É bom ressaltar que o meio reacional é aquoso. Quando a reação se realizou no tempo de $4 \mathrm{~h}$, observou-se que a banda de absorção na região da ligação O-H desaparece. Contudo, o tempo de reação escolhido para a reação de fosforilação com os demais fosfitos (fosfito de dipropila, diisopropila, diisobutila e diisopentila) foi de $4 \mathrm{~h}$, pois foi observado o decréscimo da tendência à decomposição do produto através da formação de subprodutos oriundos do aquecimento. Os compostos brutos obtidos apresentaram rendimentos variados, o copolímero de dipropila, 53,3\%, e o copolímero de diisopropila, 56,5\%, enquanto os demais apresentaram rendimentos superiores a $100 \%$, necessitando assim de etapas de purificação realizadas por cromatografia em coluna. Os copolímeros fosforilados apresentaram aspectos diferentes, sendo que o copolímero de diisopropila e o de dibutila apresentaram um aspecto rígido e os demais apresentaram um aspecto pastoso.

Em comparação com os dados relatados na literatura ${ }^{[19,20]}$ e com os resultados obtidos neste trabalho, a análise por FTIR do copolímero graftizado apresentaram-se as bandas de absorções de vibrações e deformações axiais do homopolímeo PEG6000 e do polimetacrilato de metila (PMMA). A banda em $3435 \mathrm{~cm}^{-1}$ refere-se à ligação $\mathrm{O}-\mathrm{H}$ presente no PEG 6000 e as duas bandas em $1150 \mathrm{~cm}^{-1}$ da ligação C-O (éster) e em $1722 \mathrm{~cm}^{-1}$ da ligação $\mathrm{C}=\mathrm{O}$ (éster) referem-se ao PMMA (conforme a Figura 2). 
De acordo com os dados da literatura ${ }^{[12,13,19,20]}$ os copolímeros graftizados modificados por reações de fosforilação apresentaram bandas de absorções de vibrações e deformações axiais das ligações $\mathrm{P}=\mathrm{O}$ na faixa de 1242 a $1245 \mathrm{~cm}^{-1}$, $\mathrm{P}-\mathrm{O}$ na faixa de 1104 à $1108 \mathrm{~cm}^{-1}, \mathrm{C}=\mathrm{O}$ na faixa de 1727 à $1733 \mathrm{~cm}^{-1} \mathrm{e}$ $\mathrm{C}-\mathrm{O}$ na faixa de 1241 à $1245 \mathrm{~cm}^{-1}$ (conforme a Figura 2). Sendo que os espectros dos copolímeros de diisobutila e de dipropila foram os únicos que além de apresentar as bandas de absorção referentes às ligações $\mathrm{P}=\mathrm{O}, \mathrm{P}-\mathrm{O}, \mathrm{C}=\mathrm{O}$ e $\mathrm{C}-\mathrm{O}$, apresentaram ausência da banda de absorção equivalente à ligação O-H próximo a $3400 \mathrm{~cm}^{-1}$ e à ligação $\mathrm{P}-\mathrm{H}$ próximo a $890 \mathrm{~cm}^{-1}$, que justifica a fosforilação desses dois copolímeros.

Os resultados obtidos por DSC e TGA mostraram que apenas o copolímero de diisobutila apresentou temperatura de transição endotérmica e comportamentos de degradação compatíveis a de um polímero, ou seja, com picos bem definidos e com bases mais estreitas nas transições de fusão verificados nas curvas de DSC além da tendência da estabilidade térmica manter-se quase constante a partir de determinada massa de amostra, com curvas de degradação apresentando-se em dois estágios para o copolímero e com patamares de degradação mais definidos e estáveis para as curvas de TGA, comportamentos estes característicos de moléculas de altos pesos moleculares médios (Figuras 3 e 4$)^{[20]}$.

Nas curvas de DSC dos copolímeros de dipropila, diisopropila e dibutila podem ser observados picos endotérmicos de bases largas e um deslocamento muito grande em relação à temperatura de fusão do COP GRAFT, o que não acontece em relação ao copolímero de disobutila conforme visto na Figura 3. Apenas o copolímero de isopentila mostra vários picos de transições o que pode estar relacionado ao seu perfil de degradação térmica ser instável. É possível observar também o deslocamento da temperatura de transição vítrea $(\mathrm{Tg})$, referente à cadeia principal de poli(metacrilato de metila), para os copolímeros modificados. Observa-se que o aumento do tamanho dos grupos substituintes produz uma elevação da Tg. Esse comportamento é coerente, pois a energia de ativação para o movimento desses grupos químicos é maior do que a necessária para a mobilização do grupo éster do PMMA (Figura 4) ${ }^{[21]}$. Já a diferença de conformação entre os grupos dipropila e diisopropila e os grupos dibutila e diisobutila acarretaram um deslocamento da Tg para temperaturas mais baixas pois nesse caso a diminuição da cristalinidade das amostras provocada pelo aumento do impedimento estérico é mais significativa. A curva de TGA para o copolímero de diisobutila tem perfil de degradação em dois estágios e patamares estáveis, o que condiz com moléculas de altos pesos moleculares.

Nas curvas de DSC podem ser observadas transições endotérmicas referentes à temperatura de fusão do copolímero graftizado à $50^{\circ} \mathrm{C}$ e do copolímero de diisobutila à $39^{\circ} \mathrm{C}$ (Figura 5). Essa diferença entre as temperaturas de fusão dos copolímeros se dá pela inserção de um grupo fosfito ao copolímero graftizado, grupo este muito volumoso o que dificulta as interações entre as cadeias poliméricas, acarretando na diminuição da temperatura de fusão do copolímero graftizado e conseqüentemente na modificação da estrutura e do comportamento térmico desse copolímero.

Pelas curvas de TGA verificou-se a degradação do copolímero graftizado na faixa de $250^{\circ}$ a $450^{\circ} \mathrm{C}$ e a do copolímero de diisobutila na faixa de $150^{\circ}$ a $425^{\circ} \mathrm{C}$ (Figura 6). A diferença entre as faixas de temperatura dos copolímeros se dá pelo efeito indutivo provocado pelo grupo fosfito inserido no copolímero, que diminui a força das ligações C-C do copolímero graftizado e consequentemente diminui sua temperatura de degradação, mostrando assim a modificação do mesmo.

Pela análise dos difratogramas de raios $\mathrm{X}$ dos copolímeros graftizado e de diisobutila observou-se um pequeno estreitamento dos picos de absorção referentes ao copolímero fosforilado (Figura 7). Esse pequeno estreitamento não foi suficiente para avaliar a influência na cristalinidade do copolímero após a fosforilação, embora indique uma modificação estrutural e conformacional do mesmo.

A caracterização dos copolímeros graftizado e fosforilados através das técnicas de FTIR, TGA, DSC e XRD mostrou que a fosforilação do copolímero de diisobutila foi mais efetiva.

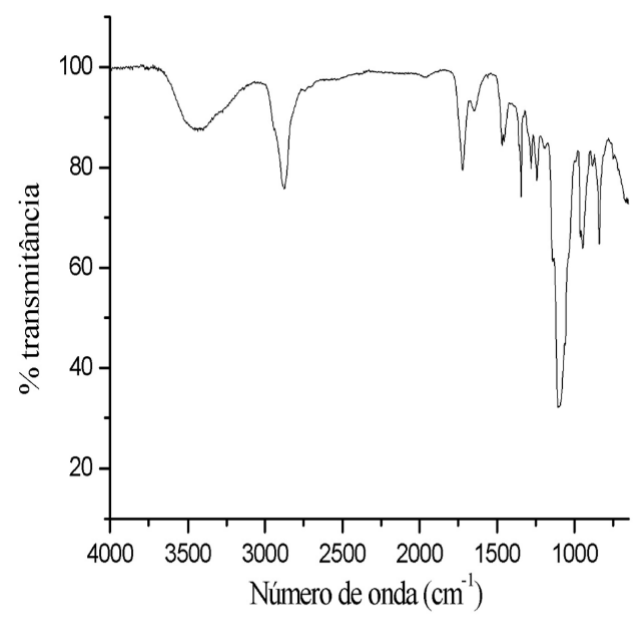

(A)

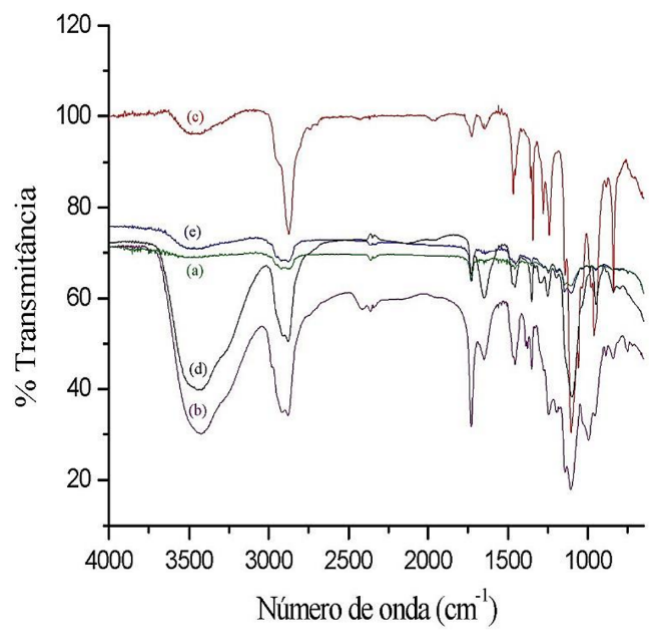

(B)

Figura 2. Espectro de FTIR do COPGRAFT (A) e dos copolímeros fosforilados (B): dipropila (a), diisopropila (b), dibutila (c), diisopentila (d), diisobutila(e), respectivamente. 


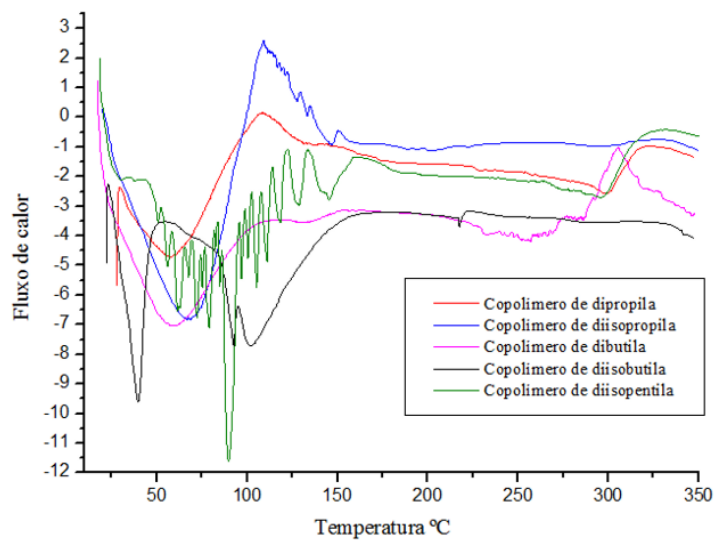

Figura 3. Curvas de DSC dos copolímeros fosforilados.

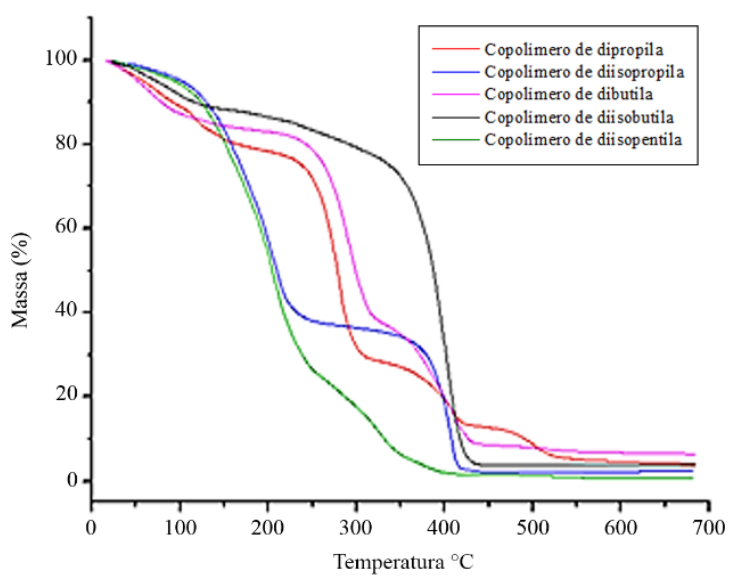

Figura 4. Curvas de TGA dos copolímeros fosforilados.

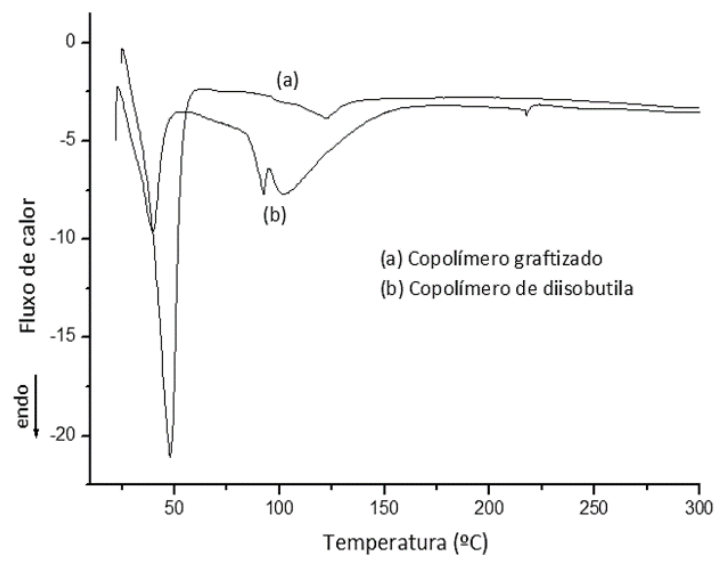

Figura 5. Curva de DSC do copolímero graftizado e do copolímero de diisobutila.

Pela técnica de GPC foi possível perceber a diferença nas massas molares do COPGRAFT, antes e depois da fosforilação. A Tabela 1 mostra os dados de massa molar numérica média $(\mathrm{Mn})$ e o índice de polidispersividade (Mw/Mn), obtido por GPC.

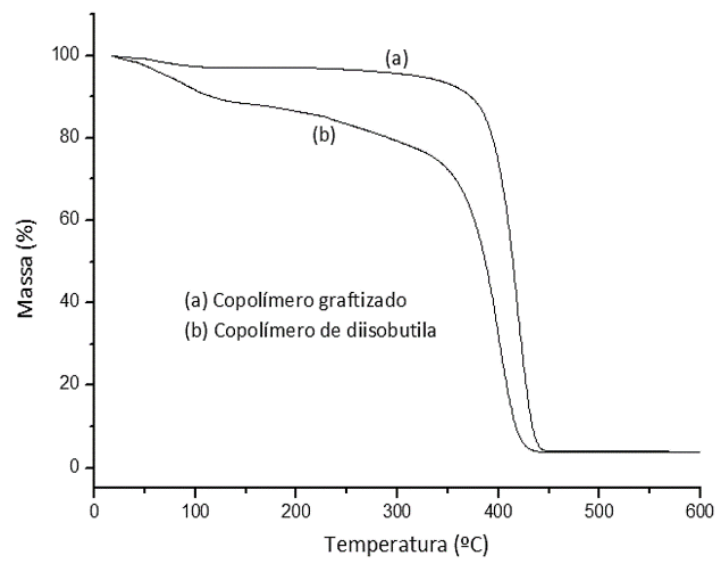

Figura 6. Curva de TGA do copolímero graftizado e do copolímero de diisobutila.

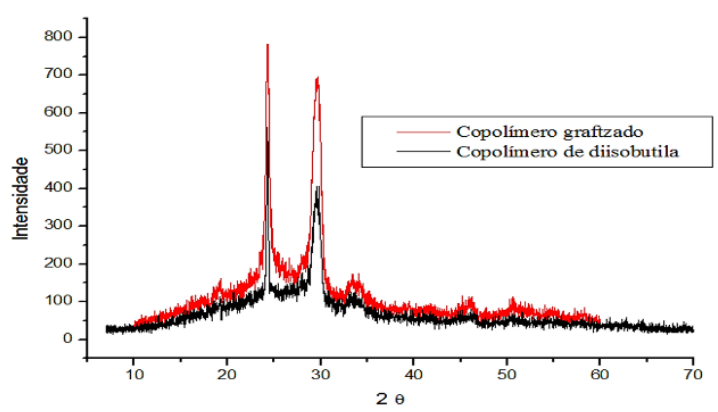

Figura 7. Difratogramas de raios $\mathrm{X}$ dos copolímeros graftizado e de diisobutila.

Tabela 1. Características moleculares dos copolímeros.

\begin{tabular}{ccc}
\hline Copolímeros & Mn $\left(\mathbf{g}^{\prime} \cdot \mathbf{m o l}^{-1}\right)$ & Mw/Mn \\
\hline COPGRAFT & 276633 & 1,46 \\
COP de diisobutila & 290306 & 1,60 \\
\hline
\end{tabular}

De modo geral foram obtidos copolímeros com elevada massa molar, na ordem de $10^{5} \mathrm{~g} \cdot \mathrm{mol}^{-1}$, e polidispersividade variando de 1,46 a 1,6, que são valores de polidispersividade comuns, encontrados na literatura, para polímeros obtidos por reação radicalar. A maior polidispersividade para o copolímero fosforilado pode estar associada à inserção de um grupo químico volumoso que conduz à formação de cadeias mais desorganizadas provocando o alargamento da faixa de distribuição de massas dos copolímeros ${ }^{[21,22]}$.

\section{Conclusões}

Na fosforilação do copolímero graftizado (COPGRAFT) é necessário um tempo reacional de $4 \mathrm{~h}$ para diminuir a decomposição do produto com o aquecimento. As técnicas aplicativas foram úteis e necessárias para a caracterização e purificação dos compostos obtidos. A reação de fosforilação foi eficiente na formação do copolímero de diisobutila e a diferença entre sua temperatura de degradação e o deslocamento da sua temperatura de transição endotérmica para temperaturas 
mais baixas quando comparada ao copolímero graftizado demonstra a modificação desse copolímero. O copolímero fosforilado terá sua atividade biológica avaliada, através da incorporação de fármacos específicos.

\section{Agradecimentos}

PROPP-UFOP/REDEMAT/UFMG/FAPEMIG.

\section{Referências}

1. Pillai, O., \& Panchagnula, R. (2001). Polymers in drug delivery. Current Opinion in Chemical Biology, 5(4), 447-451. http:// dx.doi.org/10.1016/S1367-5931(00)00227-1. PMid:11470609.

2. Oréfice, R. L., Pereira, M. M., \& Mansur, H. S. (2006). Materiais poliméricos: ciência e aplicação como biomateriais. In R. L. Oréfice, M. M. Pereira \& H. S. Mansur (Eds.), Biomateriais: fundamentos e aplicações (pp. 83-156). Rio de Janeiro: Cultura Médica.

3. Villanova, J. C. O., Oréfice, R. L., \& Cunha, A. S. (2010). Aplicações farmacêuticas de polímeros. Polímeros: Ciência e Tecnologia, 20(1), 51-64. http://dx.doi.org/10.1590/S010414282010005000009.

4. Troy, D. B., \& Beringer, P. (2006). Review: Remington: the science and practice of pharmacy. Philadelphia: Lippincott Williams \& Wilkins.

5. Gebelein, C. G., Carraher, C. E., \& Foster, V. R. (1988). Applied bioactive polymeric materials. New York: Plenum Press.

6. Zhu, Y. (2002). Properties of polymeric drug delivery systems prepared by hot-melt extrusion (Tese de doutorado). Faculty of the Graduate School, University of Texas, Austin.

7. Ferreira, V. L. S., \& Novack, K. M. (2002). Efeitos da graftização de um biopolímero na modificação da cristalinidade e decomposição de suas cadeias. In Anais do Congresso Brasileiro de Ciências dos Materiais (pp. 4975). Natal: UFRN.

8. Linhard, R. (1988). Biodegradable polymers for controlled release of drugs. In M. Rosoff (Ed.), Controlled release of drugs: polymers and aggregate system (pp. 53-85). New York: VCH Publisher.

9. Watts, P. J., Davies, M. C., \& Melia, C. D. (1990). Microencapsulation using emulsification/solvent evaporation: an overview of techniques and applications. Critical Reviews in Therapeutic Drug Carrier Systems, 7(3), 235-259. PMid:2073688.

10. Santos, V. M. R. (2003). Síntese, caracterização e o estudo da atividade biológica de novos bisfosforamidatos ebisfosforoditioatos (Tese de doutorado). Universidade Federal Rural do Rio de Janeiro, Seropédica.

11. Santos, V. M. R., Costa, J. B. N., Sant'anna, C. M. R., \& Oliveira, M. C. C. (2004). Synthesis, caracterization, molecular modeling and biological activity against artemia salina of new symmetrical. Phosphorus, Sulfur, and Silicon and the Related Elements, 179(1), 173-179. http://dx.doi. org/10.1080/10426500490257122.
12. Santos, V. M. R., Sant'Anna, C. M. R., Moya Borja, G. E., Chaaban, A., Côrtes, W. S., \& Costa, J. B. N. (2007). New bisphosphorothioates and bisphosphoroamidates: synthesis, molecular modeling and determination of insecticide and toxicological profile. Bioorganic \& Medicinal Chemistry, 35(1), 68-81. http://dx.doi.org/10.1016/j.bioorg.2006.08.006. PMid: 17055030.

13. Santos, V. M. R., Donnici, C. L., Da Costa, J. B. N., \& Caixeiro, J. M. R. (2008). Organophosphorus pentavalent compounds: history, synthetic methods of preparation and application as insecticides and antitumor agents. Quimica Nova, 30, 159-171. http://dx.doi.org/10.1590/S01004.

14. Cascone, M. G., Sim, B., \& Downes, S. (1995). Blends of synthetic and natural polymers as drug delivery systems for growth hormone. Biomaterialis, 16(7), 569-574. http://dx.doi. org/10.1016/0142-9612(95)91131-H. PMid:7492722.

15. César-Oliveira, M. A. F., Zaconcz, S., Oliveira, A. R. S., Almeida, M. C. R., Zawadzki, S. F., Akcelrud, L., Aguiar, M., Tabak, D., \& Lucas, E. F. (1999). Síntese de copolímeros metacrílicos através da modificação química do poli (Metacrilato de Metila) de massa molar controlada. Polímeros: Ciência e Tecnologia, 9(4), 156-162. http://dx.doi.org/10.1590/S010414281999000400026.

16. Novack, K. M., Sanches, N. B., \& Oliveira, C. M. F. (1993). Complexação em solução de poli (Metacrilato de Metilag-Oxido de Etileno) e Poli(Metacrilato de, Metila-g-Oxido de Propileno) com picrato de potássio. Polímeros: Ciência e Tecnologia, 3(1), 16-19. Recuperado em 12 de maio de 2015, de http://www.revistapolimeros.org.br/articles/view/ id/515c6c9e1 ef1 fae 740000557

17. Novack, K. M., Oliveira, C. \& Marize, F. (1993). Complexation of poly(methyl methacrylate-g-propylene oxide) and alkalimetal ions. Polymer Bulletin, 31, 449-456.

18. Collins, C. H., Braga, L. G., \& Bonato, P. S. (1997). Introdução a métodos cromatográficos. Campinas: UNICAMP.

19. Drummond, W. S., Wang, S. H., \& Mothé, C. G. (2004). Síntese e caracterização do copolímero poli (Ácido Lático-B-Glicol Etilênico). Polímeros: Ciência e Tecnologia, 14(2), 74-79. http://dx.doi.org/10.1590/S0104-14282004000200009.

20. Lucas, E. F., Soares, B. G., \& Monteiro, E. (2001). Caracterização de polímeros: determinação de peso molecular e análise térmica. Rio de Janeiro: Embrapa. Recuperado em 19 de maio de 2015, de http://www.bdpa.cnptia.embrapa.br

21. De Deus, J. F. (2003). Sintese e propriedades fisicas de poli(metcrilato de metila-co-metacrilato de 9-metil antracenila) (Dissertação de mestrado). Universidade Federal do Paraná, Curitiba.

22. Sperling, L. H. (2002). Introduction to physical polymer science. New York: John Wiley \& Sons.

Enviado: Dez. 03, 2013

Revisado: Nov. 07, 2014

Aceito: Jan. 09, 2015 